\title{
NARRATIVAS Y MEMORIA SOCIAL: una aproximación desde el currículo currere
}

\author{
ÁNGELA CAMILA AYALA \\ Mestre em Educação, Universidade Federal da Bahia. Faculdade de Educação - FACED \\ UFBA; Licenciada em Psicologia e Pedagogia, Universidade Pedagógica Nacional/Colômbia. \\ Professora da rede pública de ensino da Colômbia (2013-2017). Grupo de Pesquisa Formação \\ em Exercício de Professores - FEP UFBA/CNPq. ORCID: 0000-0003-1924-1907 E-mail: \\ cami9013@gmail.com
}

\section{MARIA ROSELI GOMES BRITO DE SÁ}

Professora da Universidade Federal da Bahia. Faculdade de Educação - FACED UFBA. Líder Grupo de Pesquisa Formação em Exercício de Professores - FEP UFBA/CNPq. ORCID: 00000002-5457-1074 E-mail: roselisa.rds@gmail.com 


\section{NARRATIVAS Y MEMORIA SOCIAL: na aproximación desde el currículo currer}

El presente trabajo esboza los resultados de una investigación en que pretendió abordar el proceso de enseñanza de la historia reciente, desde una perspectiva de diálogo entre las narrativas y la memoria social en el desarrollo de un currículo en "recorrido" trayendo a escena el relato del estudiante como protagonista del proceso de construcción del currículo. Se exponen puntos principales sobre el currículo escolar colombiano y como los estudiantes narran la forma en que conciben el conflicto armado del país. La narrativa estimulada en el aula, se desarrolla por medio de los aportes de currículo como currere, la experiencia como elemento generador de situaciones, es un elemento característico del proceso. Se considera este tipo de trabajos como un aporte que enriquece el campo educativo y los estudios que se realizan en esta perspectiva, además de contribuir reflexiones y anhelos que realizan los estudiantes sobre su proceso formador.

Palabras claves: Conflicto armado colombiano; Currículo currere; Memoria social; Narrativas.

\section{NARRATIVES AND SOCIAL MEMORY: an approximation from currere currículum}

The present work outlines the results of a research in which it tried to approach the teaching process of recent history, from a perspective of dialogue between narratives and social memory in the development of a curriculum in "journey" bringing to the scene the story of the student as protagonist of the process of construction of the curriculum. Main points are exposed about the Colombian school curriculum and how the students narrate the way they conceive the armed conflict in the country. The narrative stimulated in the classroom, is developed through the contributions of curriculum as currere, experience as a generator of situations, is a characteristic element of the process. This type of work is considered as a contribution that enriches the educational field and the studies carried out in this perspective, in addition to contributing reflections and desires that students make about their training process.

Keywords: Colombian armed conflict; Curriculum Currere; Social memory; Narratives.

\section{NARRATIVAS E MEMÓRIA SOCIAL: uma aproximação a partir do currículo currere}

O presente trabalho delineia os resultados de uma pesquisa na qual buscou abordar o processo de ensino da história recente, a partir de uma perspectiva de diálogo entre narrativas e memória social no desenvolvimento de um currículo em "recorrido" trazendo à cena a história da trajetória do estudante como protagonista do processo de construção do currículo. Os principais pontos são expostos sobre o currículo escolar colombiano e como os estudantes narram a maneira como concebem o conflito armado no país. A narrativa estimulada em sala de aula, é desenvolvida através das contribuições do currículo como currere, a experiência como geradora de situações, é um elemento característico do processo. Este tipo de trabalho é considerado como uma contribuição que enriquece o campo educacional e os estudos realizados nessa perspectiva, além de contribuir com reflexões e desejos que os estudantes fazem sobre seu processo de formação.

Palavras-chave: Conflito armado colombiano; Currículo currere; Memória social; Narrativas. 


\section{NARRATIVAS Y MEMORIA SOCIAL: una aproximación desde el currículo currere}

\section{Introdução}

A partir del siglo XX, Colombia se caracterizó por ser parte de los países que vivenciaron en su territorio nacional una guerra interna. Identificado por su complejidad y violencia prolongada, este fenómeno permeo la nación completa. De esta forma, en la educación, el conflicto armado es un concepto que ha sido enseñado a los estudiantes como una tragedia nacional, un evento divido en décadas, resaltando algunos acontecimientos que han sucedido en cada una de ellas, los actores violentos ocupan parte del protagonismo en la historia reciente. Las estadísticas de acciones violentas, victimas reducidas a cifras, impactos sociales y gestiones del gobierno para erradicar el conflicto armado, destacar golpes militares e incluso resaltar el trabajo de ciertas personas o condenar las acciones de otros (GONZALES, 2014), están por encima de la visibilidad que se les da a las historias de la población, esas historias que no aparecen en la narrativa oficial sobre el conflicto armado.

Vivir en medio de una guerra tiene una variación en la forma como cada persona confronta el conflicto interno, los esfuerzos por analizar continuamente la guerra en Colombia, por parte de instituciones extranjeras e instituciones colombianas, han desarrollado investigaciones en torno a sus orígenes e impactos en la sociedad. Algunas de estas investigaciones después del proceso de pos acuerdo con las FARC - EP (Fuerzas Armadas Revolucionarias de Colombia - Ejército del Pueblo) han adoptado caminos para visibilizar los procesos que permitan a la nación relatar lo sucedido. Desafío histórico para el país, la reconciliación, el perdón, un recuentro, el tejer social de la comunidad, son elementos indispensables para la reconstrucción de la memoria histórica para seguir construyendo nuestra historia. (GALINDO, RESTREPO e SANCHEZ, 2009).

La propuesta aquí presentada es un compromiso pedagógico que busca generar una reflexión en la comunidad educativa sobre los desafíos que se deben asumir para la consolidación de espacios de paz a partir de la educación, específicamente en el currículo escolar. Para ello se requiere mirar a nuevas perspectivas pedagógicas que permitan a los estudiantes alcanzar un alto nivel de compromiso con su propia formación.

Una forma para alcanzar este objetivo es la propuesta de debates y diálogos en las salas de aula, para y con los estudiantes (LOPES, 1999). Este ejercicio se puede concebir como un proceso 
más analítico y crítico del contexto violento en el cual ellos han vivido, en el cual se abordan las concepciones, juicios e ideas que los jóvenes tienen del conflicto (BECERRA, et al. 2012).

El trabajo pedagógico de carácter reflexivo, que nos interesa corresponde a dar visibilidad las narraciones sobre conflicto armado de los estudiantes que se configuran en el territorio escolar y su aproximación con el currículo escolar. La relevancia que tiene la interpretación sobre el conflicto armado en Colombia, nos lleva a detenernos a pensar y reflexionar sobre el proceso de planeación curricular, en las disciplinas escolares, en los lineamientos curriculares, en la sala de aula. Es justamente en este proceso que interesa conversar, en sus afinidades y en las resistencias con el contenido por parte del estudiante.

A partir de la perspectiva teórica de Pinar (2004), se estructura el punto de partida y al mismo tiempo se genera la inquietud, en el momento en que plantea la pregunta: ¿Cómo sería el currículo, se centráramos las disciplinas escolares en las historias y las reflexiones autobiográficas de aquellos que las soportan? Los "asuntos" en las disciplinas escolares, se refieren tanto a los asuntos humanos como a los asuntos académicos ${ }^{1}$ (PINAR, 2007 p.69). Esta pregunta se antepone como desafío y a su vez encamina el trabajo pedagógico, al cuestionar y reflexionar sobre la práctica docente, el cual se enfoca en resaltar las narrativas de los estudiantes, como producto de su experiencia y allí encontrar la convergencia con el currículo.

A partir de lo anteriormente dicho, se planteó desarrollar un ejercicio junto con los estudiantes que se centró en la producción de narrativas por parte de ellos sobre la pregunta: ¿Cuál es su concepción de conflicto armado? Con este cuestionamiento se buscó inquietar a los estudiantes para la creación de narrativas en las cuales plasmaran sus concepciones de conflicto armado, historias propias o vivencias de otros, pero sobretodo en las que se encontrase como hilo conductor el fenómeno del conflicto armado colombiano. De esta experiencia, fue posible evidenciar que algunos estudiantes han configurado su noción sobre el conflicto armado en Colombia, a través de la información construida de los medios de comunicación, otros desarrollan su concepto por las aproximaciones que llegan a realizar en lo aprendido en la sala de aula y otros tienen una influencia de las experiencias familiares o sus experiencias personales, estos son algunos factores que han marcado el concepto de este fenómeno social en los estudiantes.

\footnotetext{
${ }^{1}$ Traducción realizada por las autoras.
} 


\section{El pos acuerdo entra en la Educación}

Uno de los desafíos más importantes que la sociedad colombiana tiene actualmente es enfrentarse al pos conflicto. El pos conflicto o también conocido en Colombia como pos acuerdo se considera el periodo posterior a los tratados de paz firmados entre el gobierno de turno y a las FARC-EP. Ese proceso se presenta como una oportunidad de transformación, por eso varios sectores de la sociedad están trabajando para proponer y comenzar a actuar paralelamente al proceso que está viviendo el país. La educación presenta un aporte clave para el tránsito de la guerra para la paz, por supuesto, la escuela se torna un espacio que posibilita reconstruir los escenarios de convivencia, de dialogo, reconocimiento del otro y el respeto por la diferencia. El camino para la paz está ligado al conocimiento de la realidad nacional, al reflexionar sobre ella y proponer soluciones para la reconciliación es una "garantía de los complejos procesos que tendrá que asumir la sociedad colombiana en la etapa de pos conflicto". La catedra de la paz se concibe como una forma de responder las necesidades formativas de los estudiantes en el contexto de pos conflicto. La catedra de paz se propone, en su estructura, propiciar en las salas de aula, la reflexión y conocimiento de la historia colombiana, el dialogo de saberes, factores que permiten prácticas de reconciliación y perdón, siendo indispensable que los alumnos se apropien de este tipo de trabajo"

La catedra de la paz deberá fomentar el proceso de apropiación conocimientos y competencias relacionados con territorio, la cultura, el contexto económico y social y la memoria histórica, con el propósito de reconstruir el tejido social, promover la prosperidad general y garantizar la efectividad los principios, derechos y deberes consagrados en la Constitución. (Artículo 2, Decreto no. $1038,2015)$

Sobre el marco de la Ley no. 115 del 8 de febrero de 1194, se define currículo como el conjunto de criterios, planos de estudio, programas, metodologías y procesos que contribuyen para la formación integral y la construcción de la identidad cultural nacional, regional y local, incluyendo también los recursos humanos, académicos y físicos para colocar en práctica las políticas y desenvolver el Proyecto Educativo Institucional. La catedra de la paz se puede desarrollar en las instituciones como un plan de estudios, una temática en un área de conocimiento o por medio de proyectos de la institución. Las instituciones tienen toda la autonomía para desarrollar de la forma más pertinente ese componente (la catedra de paz) en la sala de aula.

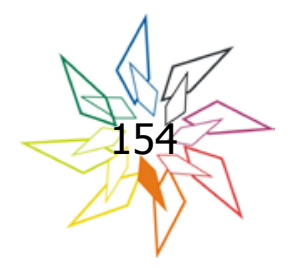


El contexto histórico, social, político, económico y cultural tiene una relación con la educación, así los procesos pedagógicos están de acuerdo como el tiempo y espacio de la sociedad. Sobre esa perspectiva comienza a buscar y concebir el currículo, los planos de estudios, los proyectos educativos, las practicas pedagógicas como otra mirada lejos de la educación tradicional, para trabajar en procesos de transformación de enseñanza y aprendizaje más dinámicos y coherentes con la mudanza social.

La memoria histórica y pedagógica se constituye como una herramienta de abordaje indispensable como dispositivo pedagógico (ZULUAGA e MARIN, 2006) de gran valor en la tarea de entender y abordar como han surgido las concepciones y narrativas de conflicto armado nacional y otros factores relacionales (BETANCOURT, 2004) Para tal finalidad se hace necesaria una perspectiva que concentre los esfuerzos de la comunidad educativa, para mejorar la forma en que se da la adaptación de la escuela en un contexto de violencia y abusos a los derechos más fundamentales de los seres humanos (RODRÍGUEZ, S. P. Y SÁNCHEZ, 2009a, 2009b).

El sistema educativo colombiano precisa de una propuesta educativa más vigente y pertinente frente a las dificultades nacionales, y dentro de las salas de aula del país es posible encontrar un camino para dar una garantía de vivir en paz en un país que pueda superar el conflicto y reconstruirse.

\section{Las narrativas y el currículo currere}

La narrativa en este proceso de investigación es utilizada en tres categorías: la primera configura la narrativa como dispositivo formativo, en este aspecto se entrecruzan la memoria, la narrativa y la educación, mediadas por la experiencia del estudiante. Para Brunner (2004) el pensamiento narrativo, es visto como la capacidad de narrar sobre sí mismos, en este sentido las narrativas conducen a los estudiantes a reconocerse como sujetos en formación. De esta forma, se interpretan los procesos que los estudiantes configuran e incorporan todas las experiencias de aprendizaje que tienen elaborado, dando mayor visibilidad al conocimiento del estudiante mediado por su experiencia. A partir de las interpretaciones por medio de la memoria, que traen los relatos de los estudiantes, se abre el camino para adentrarse en la discusión sobre el currículo como currere, por medio de la narrativa como metodología para desarrollar las actividades curriculares. El aspecto metodológico también se caracteriza la tercera dimensión concedida a la narrativa, de esta vez refiriéndose al propio camino metodológico adoptado para la realización de esta investigación.

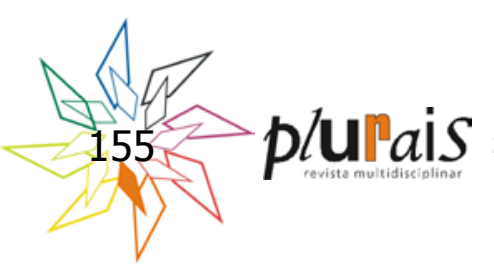


El pensamiento narrativo es el instrumento capaz de captar las vicisitudes de la intencionalidad humana, siendo que los seres humanos tienen identidad porque son capaces de narrar historias sobre ellos mismos. A partir de esta perspectiva, es a través de las narraciones que podemos dar significado a nuestra vida y comprender los otros, que las conduce a entender nuestro actuar e interpretar las acciones de los demás.

Las narrativas toman el formato del relato que permite comprender no solo o familiar, sino también lo extraño o aquello que se distancia de lo esperado, de lo normativo. También se puede decir que el pensamiento narrativo tiene un importante componente educativo y cultural, en la medida en que el objetivo de la educación es ayudar a encontrar nuestro camino dentro de nuestra cultura y comprenderla en las complejidades y contradicciones.

En este punto, la narrativa opera entre la privacidad del sujeto y el espacio de lo socio histórico de su existencia, sea ampliando la comprensión de los fenómenos sociales y grupales, o sea haciendo emerger un nueva mirada sobre sí mismo (CARVALHO, 2003). Partiendo del punto de vista de Chagas (2007), cuando se abordan las narrativas dentro de la sala de aula, se tiende a penetrar la "caja negra" escolar, dando relevancia a las vivencias, experiencias e historias que los sujetos traen consigo para la sala de aula y que hacen parte del cotidiano escolar de ellos. "Penetrar la "caja negra" escolar, abordando los dispositivos de organización y la cotidianidad en sus prácticas, poner en escena la perspectiva de los agentes educacionales" (CHAGAS, p. 93, 2007).

A partir de este referencial, en esta pesquisa las narrativas son consideradas piezas clave en la hora de indagar sobre los procesos que tienen lugar en la construcción del concepto del conflicto armado, pudiendo traer posibles soluciones a la ausencia del debate sobre el conflicto, sobre las propuestas nacionales de parar con el conflicto armado; además de eso pueden permitir explorar la forma como la sociedad colombiana entiende y experimenta vivencialmente el conflicto a partir tanto de lo corporal como de lo sensorial, hasta lo lingüístico, y como este se ve reflejado en la esfera de la memoria social (GALINDO, RESTREPO e SANCHES, 2009).

El relato, la narrativa, el cuento, la historia y todas las formas orales y escritas vistas como una propuesta de abordaje a partir de las cuales se pueden levantar las experiencias sobre el conflicto, es un proceso continuo de intercambio y discusión. Son formas validas de abordarlo, formas en las cuales tanto el estudiante que narra, como el profesor que escucha o lee (CARVALHO, 2008; HUERTA, 1995), participen en la construcción o recreación de un momento, de una visión, de un pasado y un presente. 
Tratándose apenas de una aproximación que considera pertinente a la elaboración continua de reflexiones que provocan el dialogo y el intercambio de narrativas, historias y anécdotas, elementos indispensables en la construcción de una perspectiva común, que pueda atacar directamente los efectos negativos del conflicto armado en Colombia.

Por ultimo Paiva (2018) basada en Gennete, nos aporta que existe una enorme complejidad desarrollada en el proceso de representar algo, alguien o alguna cosa. Gennette (2009) define narrativa como la representación de un acontecimiento o de una serie de acontecimientos, reales o de ficción, por medio del lenguaje e, mas particularmente, del lenguaje escrito. En este sentido, se tiene en cuenta que todo lo que puede ser comprendido es lenguaje. Toda la comprensión se deriva de las posibilidades que se tienen de representar de manera verbal o no. Roland Barthes (2008) aporta para este trabajo la propuesta de tres niveles de descripción para la obra narrativa: el nivel de las funciones, o de las acciones y de la narración.

Estos tres niveles están ligados entre si según una integración progresiva: una función solo tiene sentido si se ubica en la acción general de un actante; y esta acción misma recibe su sentido último del hecho de que es narrada, confiada a un discurso que es su propio código (BARTHES, 2009, p.15).

En esta perspectiva la acción a ser interpretada en las narrativas de los estudiantes, que se refieren al conflicto armado en Colombia, trayendo más referencias para la comprensión de este fenómeno descrito teóricamente.

\section{Memoria como dispositivo de trabajo pedagógico}

Imagen 1. Título: Conflicto Armado en Colombia.

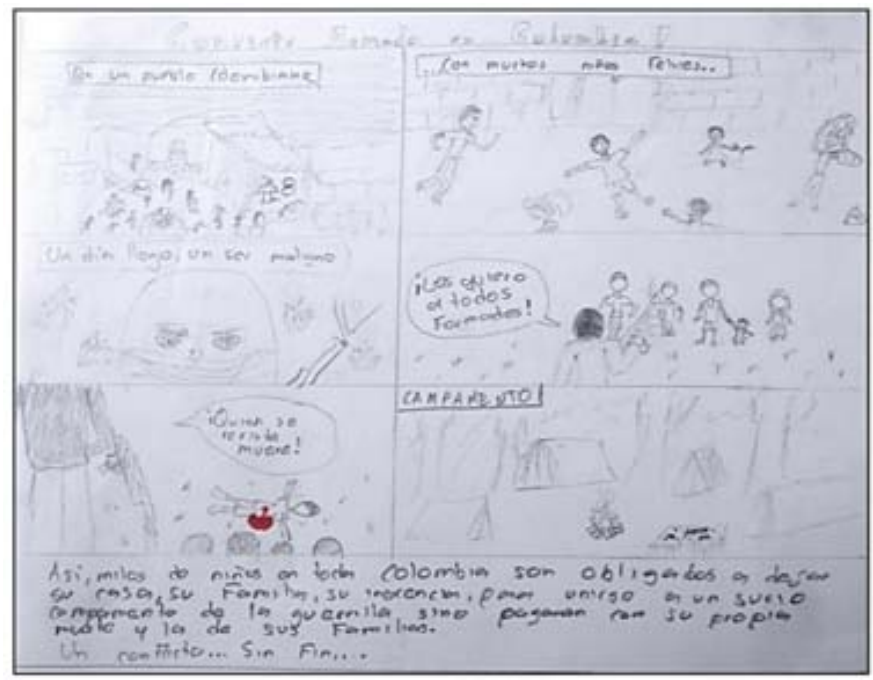

Fonte: Joaquín, 15 años. 
La sala de aula es un contexto vivo, en donde los estudiantes son protagonistas del currículo, esta herramienta puede llevar a generar acciones transformadoras en el sistema educativo. Enseñar historia reciente, unificada con la memoria colectiva, es un instrumento que contribuye a la enseñanza, como fortaleza para reflejar la pluralidad de experiencias que convergen en la sala de aula, convirtiéndose aún más significativa para la construcción de conocimiento. El trabajo en aula con los estudiantes utilizando la memoria como dispositivo de trabajo pedagógico, puede tener alcances en la reflexión sobre el contenido curricular y la cotidianidad de los estudiantes.

Imagen 2. Título: Lo que calla un país.

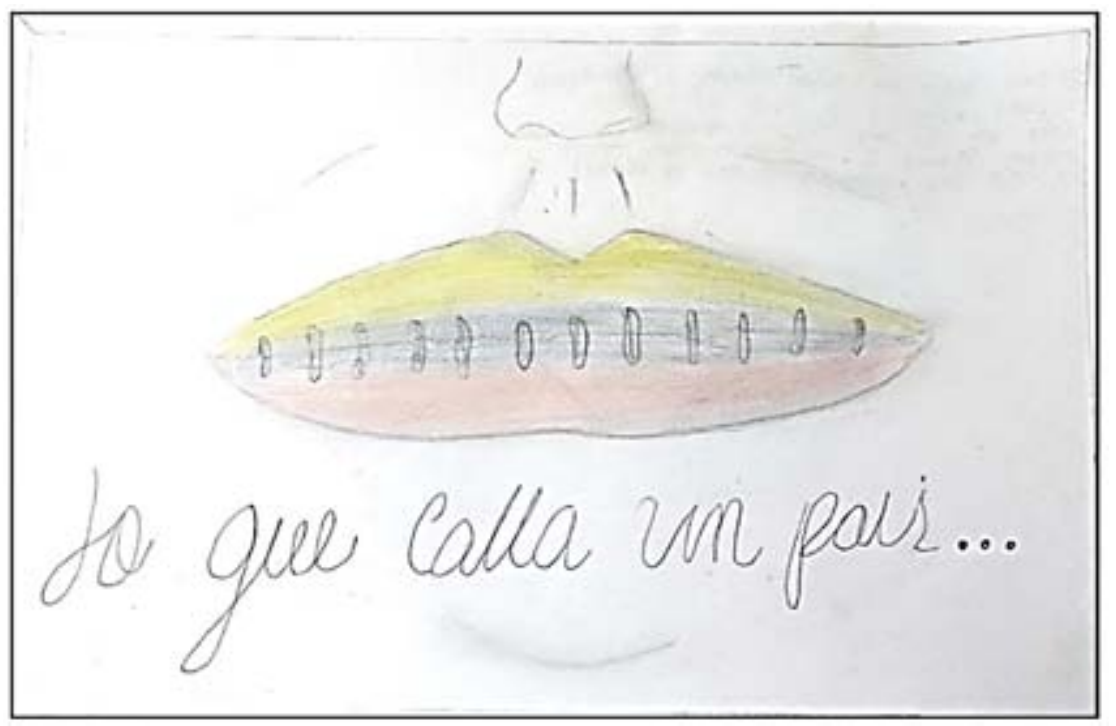

Fonte: Juliana, 14 años

Además, las historias cargadas de recuerdos, de emociones, de anécdotas, de dolor y hasta de esperanza, revelan como el estudiante construye su experiencia y logra describirla por medio de una narrativa. La memoria colectiva tiene un papel el cual permite relatar cómo ha sido elaborado el conflicto por los estudiantes, el interés es rescatar esas experiencias por medio de un ejercicio de memoria. 
"Yo y mi familia, si nos podemos considerar victimas porque hace 20 años mataron a mi bisabuela [...] Los paramilitares la mataron a ella porque decían que era una bruja, y mi mamá y mi tía tuvieron que salir del pueblo, porque todos las estaban buscando para matarlas." Juan Diego, 14 años.

De acuerdo con Sá (2010), esto trae un elemento importante siendo un recurso formativo, para todos los involucrados en la educación escolar. Es indispensable el estudio del currículo para los educadores y para todos aquellos que están implicados sin importar el vínculo que tengan. El resultado de eso será una contribución para el recurso formativo de los estudiantes. El estudiante como actor del currículo produce nuevas prácticas y aprendizajes (MACEDO, 2007). El estudiante como actor del currículo y dentro del mismo, debe ser tenido en cuenta la importancia de las diferencias, sean culturales o de clase, pero que llevan al estudiante como actor del currículo y dentro del currículo.

“... ahora lo que necesitamos es un país en donde de verdad haya paz, donde la gente pueda vivir tranquila, sin temor a que sean sacados de sus tierras, donde tengan miedo de que les quitan sus hijos de las manos. Necesitamos una Colombia en paz y el cambio empieza por mí, con mi tolerancia, con mi respeto, con mi paciencia, yo marco la diferencia en este país que necesita paz." Estefanía, 15 años.

La experiencia pedagógica toma como hipótesis que, en cualquier momento, él, o ella, está en una situación biográfica "(PINAR y GRUMET, 1976 apud PINAR, 2007), es decir, que él, o ella, se sitúa en un tiempo histórico y contexto cultural, pero en una forma significante singular, una situación a ser expresada en la voz autobiográfica de cada uno (PINAR, 2007, p.66). Por lo tanto, se indaga generar en el trabajo de aula, generar una reflexión con la comunidad educativa sobre los desafíos que se deben asumir para la consolidación de espacios de paz a partir de la educación y el papel del currículo en el pos acuerdo. Para eso es significativo que los estudiantes alcancen un nivel de compromiso con su formación académica. "¿Por qué no y el currículo escolar una provocación para que los alumnos reflexionen sobre ella y piensen críticamente sobre ellos mismos y el mundo que van a heredar?"

El propósito educativo del currículo de la escuela pública es comprender, comprender las relaciones entre el conocimiento académico, el estado de la 
sociedad, los procesos de auto-formación y el carácter del momento histórico en que vivimos, en el cual otros vivieron y en el que nuestros descendientes van a vivir un día ${ }^{2}$. (PINAR, 2007, p.292).

La noción del currículo como currere, proviene del trabajo de William Pinar, teórico del currículo que inventa el método currere para sustentar sus estudios de la auto reflexión dentro del proceso educativo. Pinar destaca que la forma latina del infinitivo del termino currículo significa recorrer el camino, lo que proporciona una estrategia para que los interesados en estudiar currículo, trabajen las relaciones entre el conocimiento académico y las historias de vida. Pinar, denomina cuatros momentos en el método currere: progresivo, analítico, sintético y regresivo, esos elementos son importantes para entrar en los estudios autobiográficos y la experiencia pedagógica (PINAR, 2007).

Con base a lo anteriormente mencionado y teniendo en cuenta que en el currículo currere, el termino currere, está en la etimología misma del termino currículo, es importante destacar que la propuesta se denomina como una metodología de actuación docente que sustenta el ejercicio sistemático de la auto reflexión dentro del proceso educativo (PINAR, 2004); fornece una estrategia para los alumnos del currículo estudien las relaciones entre conocimiento académico e historia de vida, en el interés de la auto comprensión y reconstrucción social (MONTOYAVARGAS, 2014).

De acuerdo con Sá (2010), esto trae un elemento importante siendo un recurso formativo, para todos los involucrados en la educación escolar. Es indispensable el estudio del currículo para los educadores y para todos aquellos que están implicados sin importar el vínculo que tengan. El resultado de eso será una contribución para el recurso formativo de los estudiantes. El estudiante como actor del currículo produce nuevas prácticas y aprendizajes (MACEDO, 2007). El estudiante como actor del currículo y dentro del mismo, debe ser tenido en cuenta la importancia de las diferencias, sean culturales o de clase, pero que llevan al estudiante como actor del currículo y dentro del currículo.

Por lo tanto, se incluye en el trabajo de aula, una reflexión con la comunidad educativa sobre los desafíos que se deben asumir para la consolidación de espacios de paz a partir de la educación y el papel del currículo en el pos acuerdo. Para eso es significativo que los estudiantes alcancen un nivel de compromiso con su formación académica.

${ }^{2}$ Traducción realizada por las autoras.

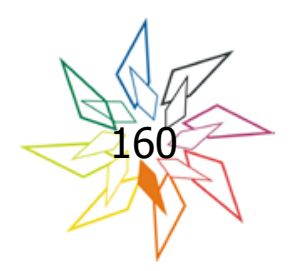


El propósito educativo del currículo de la escuela pública es comprender las relaciones entre el conocimiento académico, el estado de la sociedad, los procesos de auto-formación y el carácter del momento histórico en que vivimos, en el cual otros vivieron y en el que nuestros descendientes van a vivir un día (PINAR, 2007, p.292).

\section{Consideraciones finales}

Enseñar historia reciente fusionándola con la memoria colectiva es una herramienta que contribuye a la enseñanza, es una de sus mayores fortalezas porque en la pluralidad de experiencias, se torna aún más significativo la construcción de conocimiento. "el estudioso el método currere imagina futuros posibles" (PINAR, p.67). Además, que el maestro logra tensionar la "historia oficial" aquella que hace parte de los lineamientos de la disciplina y la memoria social, logrando experiencias significativas de trabajo en el aula.

El trabajo en el aula con los estudiantes usando la memoria como dispositivo de trabajo pedagógico puede reflejar sobre el contenido curricular, y la cotidianidad de los estudiantes, historias con subjetividad cargadas de recuerdos revelando como el estudiante reconstruye su experiencia y la describe en su narrativa. La memoria colectiva juega un papel que nos relata cómo ha sido elaborado el conflicto por los estudiantes. El interés es rescatar la experiencia del alumno por medio de la memoria.

\section{REFERÊNCIAS}

ALCALDÍA MAYOR DE BOGOTÁ, D.C. Decreto 1038 Por el cual se reglamenta la Cátedra de la Paz, 2015.

BECERRA, A. (et al.). Memoria, conflicto y escuela: voces y experiencias de maestros y maestras en Bogotá. Instituto para la Investigación Educativa y el Desarrollo Pedagógico. Instituto para la Investigación Educativa y el Desarrollo Pedagógico, IDEP. 2012.

BARTHES, R. (et al.). Análise estrutural das narrativas. 5 ed. Petrópolis. Vozes, 2008.

\footnotetext{
${ }^{3}$ Traducción realizada por las autoras.
} 
BERCERRA, J, ACEVEDO, R, CORTÉS, R. Escuela, memoria y conflicto en Colombia. Un ejercicio del estado del arte de la temática. Revista Colombiana de Educación, n. 62, Primer semestre de 2012, Bogotá, Colombia. 2012.

BETANCOURT, D. E. Memoria individual, memoria colectiva y memoria histórica. Lo secreto y lo escondido en la narración y el recuerdo. La práctica investigativa en ciencias sociales. UPN Universidad Pedagógica Nacional: Bogotá, 2004

BRUNER, J. Life as a narrative. Social Research, 71(3), 691-710. 2004.

CARVALHO, I. C. M. Biografia, identidade e narrativa: elementos para uma análise hermenêutica. Horizontes Antropológicos, Porto Alegre, v. 9, n. 9, p.283-302, jul. 2003.

CHAGAS, M. M. Manuales de pedagogía, materialidad de lo impreso y circulación de modelos pedagógicos en el Brasil. Revista Colombiana de Educación, 52, 92-113. 2007

GALINDO, H; RESTREPO, J. A.; SANCHEZ, F. Conflicto y pobreza en Colombia: un enfoque institucionalista. In: RESTREPO, J. A. y APONTE, D. (Eds.) Guerra y violencias en Colombia Herramientas e interpretaciones. Editorial Pontifícia Universidad Javeriana. Bogotá, 2009.

GENETTE, G. Fronteiras da Narrativa. In: BARTHES, R. (et al.). Análise estrutural das narrativas. 6 ed. Petrópolis. Vozes, 2009.

GONZALES, M. I. C. La violencia contada a los escolares. Conflicto social y memoria en los manuales educativos del siglo XX. Análisis político no 81, Bogotá, mayo-agosto, 2014: págs. 3248.

LOPES, A. R. C. Pluralismo cultural em políticas de currículo nacional. In: MOREIRA, A. F. B. (Org.). Currículo: políticas e práticas. Campinas: Papirus, 1999. p. 59-80.

MACEDO, R. S. Currículo: Campo, Conceito e Pesquisa. Editora Vozes, 2007.

MONTOYA-VARGAS, J. Curriculum Studies in Colombia. In: PINAR, W. F. (Org.) International Handbook of Curriculum Research. 2nd Edition, Taylor \& Francis, 2014.

MEN. Ley $N^{\circ} 1732$ del 1 de septiembre de 2014. "Por la cual se establece la cátedra de la paz en todas las instituciones educativas del país”. 2014. 
PINAR, W. Currere: aquel primer año. Investigación Cualitativa, 2(1) pp. 55-65, 2017. DOI: http:// dx.doi.org/10.23935/2016/01035. Disponível em: https://ojs.revistainvestigacioncualitativa.com/ index.php/ric/article/view/59. Acesso em janeiro de 2018.

PINAR, W. F. O que é a Teoria do Currículo? Porto Editora. 2007.

PINAR, W. F. What's Theory of Curriculum? y Lawrence Erlbaum Associates, 2004.

RODRÍGUEZ, S. P.; SÁNCHEZ, O. Problemáticas de la enseñanza de la historia en Colombia: trabajar con la memoria en un país en guerra. Revista Reseñas, No 7. Argentina: Apehu. (2009 a).

RODRÍGUEZ, S. P; SÁNCHEZ, O. Narrativa, memoria y enseñanza del conflicto armado colombiano: propuestas para superar las políticas del olvido y la impunidad. En: Adrián Serna (Compilador). El papel de la memoria en los laberintos de la justicia, la verdad y la reparación. Bogotá: Ipazud, U. Distrital. (2009 b).

SÁ, M. R. G. e ALVES, I. C. P. Narrativas curriculares em currículos de cursos de formação de professores em exercício. In: GALEFFI, D. TOURINHO, M.A. SÁ, M.R.G.B. (Orgs.) Educação e Difusão do Conhecimento: caminhos da formação. 1ed. , v.1, p. 405-432. Salvador, EDUNEB, 2016.

SÁ, M. R. G. Currículo e Formação: atualizações e experiências na construção de existências singulares. In: SÁ, M. R. G; FARTES, V. L. B. (Orgs). Currículo, formação e saberes profissionais: a (re) valorização epistemológica da experiência. Salvador: EDUFBA, 2010. p. 37-61.

SÁ, M. R. G. Pontos sobre Currículo Escolar. Material didático utilizado para estudos sobre Currículo nos cursos de Graduação. Universidade Federal da Bahia, Faculdade de Educação, Departamento de Educação I, 2008.

SANTOS, A. S; LAURO, B. R; MATTOS, G. F; SANTIAGO, M. C. Memórias e narrativas: múltiplos olhares na formação reflexiva. Revista da FAEEBA - Educação e Contemporaneidade, Salvador, v. 17, n. 29, p. 169-174, jan./jun., 2008.

VIDAL, R. et. al. Efectos del desplazamiento interno en las comunidades de las zonas de recepción. Estudio de caso en Bogotá, DC Colombia en las localidades de Suba y Ciudad Bolívar. Comité Nacional de la Cruz Roja Colombiana. Publicado por: Proyecto del Instituto BrookingsLondon School of Economics sobre el Desplazamiento Interno Bogotá, 2011. 38 p.p.

ZULUAGA O. L. y MARÍN D, D. L. Memoria colectiva: memoria activa del saber pedagógico. Revista Educación y Ciudad. Bogotá, D.C. IDEP. 2006. 